\title{
Hyperactivation of B-Type Motor Neurons Results in Aberrant Synchrony of the Caenorhabditis elegans Motor Circuit
}

\author{
Yingchuan B. Qi, ${ }^{1,2}$ Michelle D. Po, ${ }^{3,4}$ Patrick Mac, ${ }^{5}$ Taizo Kawano, ${ }^{3}$ Erik M. Jorgensen, ${ }^{5}$ Mei Zhen, ${ }^{3,4}$ and Yishi Jin ${ }^{2}$ \\ ${ }^{1}$ Institute of Developmental and Regenerative Biology, School of Life and Environmental Sciences, Hangzhou Normal University, 310036 Hangzhou, China, \\ ${ }^{2}$ Howard Hughes Medical Institute, Neurobiology Section, Division of Biological Sciences, University of California, San Diego, La Jolla, California 92093, \\ ${ }^{3}$ Samuel Lunenfeld Research Institute, Mount Sinai Hospital, Toronto, Ontario M5G 1X5, Canada, ${ }^{4}$ Department of Molecular Genetics, University of \\ Toronto, Toronto, Ontario M5S 1A8, Canada, and ${ }^{5}$ Howard Hughes Medical Institute, Department of Biology, University of Utah, Salt Lake City, Utah 84112
}

Excitatory acetylcholine motor neurons drive Caenorhabditis elegans locomotion. Coordinating the activation states of the backwarddriving A and forward-driving B class motor neurons is critical for generating sinusoidal and directional locomotion. Here, we show by in vivo calcium imaging that expression of a hyperactive, somatodendritic ionotropic acetylcholine receptor ACR-2(gf) in A and B class motor neurons induces aberrant synchronous activity in both ventral- and dorsal-innervating B and A class motor neurons. Expression of ACR-2(gf) in either ventral- or dorsal-innervating B neurons is sufficient for triggering the aberrant synchrony that results in arrhythmic convulsions. Silencing of AVB, the premotor interneurons that innervate B motor neurons suppresses ACR-2(gf)-dependent convulsion; activating AVB by channelrhodopsin induces the onset of convulsion. These results support that the activity state of B motor neurons plays an instructive role for the coordination of motor circuit.

\section{Introduction}

The complete wiring diagram of the nematode Caenorhabditis elegans nervous system offers an advantage to examine general principles of neural network operation. The C. elegans locomotion behavior is derived from multiple components, including the neuromuscular system, the convergence of sensory signals, and the internal physiological state (de Bono and Maricq, 2005; Bargmann, 2012). At the core of the motor circuit are five classes of acetylcholine motor neurons (VB, DB, VA, DA, and AS) that directly innervate body wall muscles (White et al., 1976; Chalfie et al., 1985; Richmond and Jorgensen, 1999). These motor neurons excite body muscles to generate sinusoidal bends in the dorsal-ventral plane, which are then propagated from head to tail to drive forward locomotion.

Received Aug. 21, 2012; revised Jan. 23, 2013; accepted Feb. 5, 2013.

Author contributions: Y.B.Q., M.D.P., M.Z., and Y.J. designed research; Y.B.Q. and M.D.P. performed research; P.M., T.K., and E.M.J. contributed unpublished reagents/analytic tools; Y.B.Q., M.D.P., M.Z., and Y.J. analyzed data; Y.B.Q., M.Z., and Y.J. wrote the paper.

This work was supported by National Institutes of Health Grants 5R01NS035546 (Y.J.) and 5R01NS034307 (E.M.J.), Canadian Institutes of Health Research Grants MOP74530 and 93619 (M.Z.), and National Natural Science Foundation of China Grant NSFC31171197 (Y.B.Q.). The work by Y.B.Q. was mainly performed at the University of California, Division of Biological Sciences, Neurobiology Section, where he was a research scientist supported by National Institutes of Health Grant NS035546 awarded to Y.J., M.D.P. was supported by a Natural Sciences and Engineering Research Council of Canada postgraduate fellowship. Y.J. and E.M.J. are investigators of Howard Hughes Medical Institute. We thank C. Giurumescu for help in light control device, Z. Wu for imaging analysis, and A. D. Chisholm and laboratory members for discussions.

The authors declare no competing financial interests.

Correspondence should be addressed to Yingchuan B. Qi, Institute of Developmental and Regenerative Biology, School of Life and Environmental Sciences, Hangzhou Normal University, 310036 Hangzhou, China. E-mail: billyqi@idrbio.org.

DOI:10.1523/JNEUROSCI.4017-12.2013

Copyright $\odot 2013$ the authors $\quad 0270-6474 / 13 / 335319-07 \$ 15.00 / 0$
Despite an extensive understanding of the development and synaptic physiology of the motor neurons, mechanisms underlying the motor coordination remain not well understood. Early studies suggest that alternating contraction of dorsal and ventral body muscles driven by the acetylcholine motor neurons may partially depend on cross-inhibition from the GABA motor neurons (Walrond and Stretton, 1985; White et al., 1986; McIntire et al., 1993). The long undifferentiated axonal processes of the $\mathrm{A}$ and $\mathrm{B}$ class motor neurons have also been postulated to serve as stretch feedback (White et al., 1986). A recent study suggests that the B motor neurons are activated during stretch (Wen et al., 2012). The interneuron DVA was found to modify the amplitude of body bends (Li et al., 2006). Innexin-based gap junctions play critical roles for maintaining an imbalanced activity of the A and B classes of acetylcholine motor neurons, and the imbalance drives the directionality of movement (Kawano et al., 2011).

Previously, we reported an acetylcholine receptor ACR-2 that is expressed in four classes of acetylcholine motor neurons (VB, DB, VA, and DA). Loss of function of ACR-2 leads to a reduced velocity and a decreased frequency of acetylcholine miniature postsynaptic current (mPSC) at the neuromuscular junctions (NMJs) (Jospin et al., 2009). A gain of function mutation in ACR-2(gf) causes increased mPSC and a severe disruption of undulation accompanied by seizure-like convulsions. Here, we show that ACR-2 is localized to soma and dendrites of the acetylcholine motor neurons. ACR-2(gf)expression in B class motor neuron specifically upregulates their activity state and drives the motor circuit to aberrant synchrony. The input premotor interneuron AVB has an in- 
structive effect on the B neuron activity state to regulate locomotion pattern.

\section{Materials and Methods}

Genetics. Hermaphrodites of the following strains were used in this study: EG5687: unc-119(ed3); Pacr-2::acr-2:::ffp(oxSi39); CZ11321: acr-2(n2420); Pacr-2::acr-2::gfp(oxSi39); CZ11991: Pitr-1 pB-acr-2::gfp(juEx2726); CZ13763: Pacr-2-acr-2::gfp(juSi21); CZ13764: acr-2(ok1887); Pacr2-acr-2::gfp(juSi21); CZ12455: Pacr-2-acr-2(n2420)::gfp(juSi19); CZ12456: acr-2(ok1887); Pacr-2-acr-2(n2420)::gfp(juSi19); CZ10402: acr-2(n2420); ZM4898: Pacr-2-D3cpv(hpIs171); ZM5567: acr-2(n2420); Pacr-2D3cpv(hpIs171); CZ11599: acr-2(ok1887); Pacr-2-acr-2(n2420):: gfp(juEx2585); CZ12226: Pdel-1-acr-2(n2420)::gfp(juEx2817); CZ13327: Punc-129-acr-2(n2420)::gfp(juEx3239); CZ13290: Pacr-5-acr-2(n2420):: gfp(juEx3228); CZ13285: Punc-4-acr-2(n2420)::gfp(juEx3223); CZ13621:

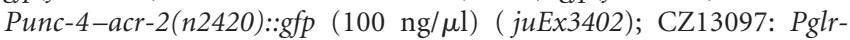
1-acr-2(n2420)::gfp(juEx3155); CZ12961: Pmyo-3-acr-2(n2420):: gfp (juEx3111);ZM3087: unc-9(fc16) unc-7(e5); CZ14383: acr-2(n2420) unc9(fc16) unc-7(e5); BLW47: unc-9(fc16) unc-7(e5); Pacr-5-acr-2(n2420):: gfp (ybqEx11); BLW37: unc-9(fc16) unc-7(e5); Punc-4-acr-2(n2420):: gfp(juEx3402); BLW24: acr-2(n2420); Psra-11-TeTx::mCherry(ybqEx1); ZM5694: acr-2(n2420); Psra-11-twk-18(gf)-UrSL-wCherry(hpEx2362); CZ16469: acr-2(n2420); Psra-11-ChR2::yfp(juEx4768).

Molecular biology. To create the MosSCI construct for oxSi39, acr-2::gfp was subcloned from pSC349 into a pENTR Gateway vector (Invitrogen) and then recombined into pCFJ201 (for insertion at the cxTi10882 site) along with acr-2 promoter and unc-54 3'UTR sequences. acr- $2 \mathrm{cDNA}$ was generated by PCR from pSC371 and cloned into pENTR vector. GFP was amplified from pPD95.75 and inserted into acr-2 cDNA at AccIII site. acr-2(gf-V309M) was made by site-directed mutagenesis. The promoter constructs were cloned by PCR from genomic DNA into pENTR: Pitr-1 $(p B)$, 2318 bp; Pdel-1, 1972 bp; Punc-129 (neuron), 2642 bp; Punc-4, 2536 bp; Pacr-5, 4320 bp; Pacr-2, 1966 bp; Pglr-1, 772 bp. A pENTR vector containing Psra-11 (promoter A; 4550 bp) was subcloned from pOH149 (O. Hobert, Columbia University, New York, NY). A pDEST Gateway construct containing tetanus toxin $(\mathrm{Te} T \mathrm{~T}$ ) was derived from pEM10 (C. Bargmann, Rockefeller University, New York, NY). A pENTR vector containing ChR2::YFP was subcloned from Pmyo-ChR2::YFP (A. Gottschalk, Goethe University Frankfurt, Frankfurt, Germany). Details of constructs are available on request.

Whole-mount immunostaining and image processing. The procedures were described previously (Van Epps et al., 2010). The antibodies used in this study include the following: rabbit anti-GFP (A11122; Invitrogen), 1:500; and anti-UNC-17 MAb1403 (J. Rand, Oklahoma Medical Research Foundation, Oklahoma City, OK), 1:100.

To analyze colocalization of anti-GFP (red) and anti-UNC-17 (green) signals, the monochromic images were analyzed in MATLAB (MathWorks). For each pixel point on the $x$-axis (along the cord), the positions for peak intensity of red and green fluorescence along the $y$-axis (perpendicular to the cord) were measured and plotted. The average distance between red and green center lines was calculated for each image (500800 pixels of the cord, $140 \mathrm{~nm} /$ pixel), and averaged data were presented ( $n=6$ images).

To analyze the relative fluorescence intensity of Pacr-2-acr-2::gfp (juSi21) and Pacr-2-acr-2(gf)::gfp (juSi19), animals were stained and images were processed as described above. The intensity for red (anti-ACR-2::GFP) or green (anti-UNC-17) signals were measured using MetaMorph (Molecular Devices). After subtracting background signals, the red/green ratios were plotted in GraphPad Prism (GraphPad Software).

Calcium imaging and data analyses. L4 animals carrying Pacr-2-D3cpv ( $h$ PIs171) were imaged and analyzed as described previously (Kawano et al., 2011). Periods of convulsions were visually identified by simultaneous contractions along the segment of the recorded nerve cord. The cross-correlation between VB9 and DB6 signals was calculated using MATLAB, with lags of $\pm 15 \mathrm{~s}$.

Quantification of convulsion rate. The quantification of convulsion behaviors was described previously (Jospin et al., 2009).

Analysis of convulsion behavior induced by channelrhodopsin activation. The L4 animals were transferred onto all-trans retinal (ATR) or control plates (no ATR) and cultured overnight. Individual animals were transferred to a non-seeded nematode growth medium plate. The shutter that controls blue light was open for $50 \mathrm{~ms}$, followed by $4950 \mathrm{~ms}$ delay. Animals were illuminated through $5 \times$ objective under the Carl Zeiss M2 microscope and were video-recorded using Carl Zeiss AxioCam MRm at 10 frames/s.

The videos were imported into NIH ImageJ. Each frame was thresholded to extract the binary image of the body. The body area was normalized against the frame immediately before the application of blue light stimulation. Each data point represents the mean normalized body area at a given time point from 5 to 12 blue light stimulation events.

\section{Results}

\section{ACR-2 localizes to dendrites and soma of the acetylcholine A and $B$ motor neurons}

We analyzed the subcellular compartment of ACR-2 by generating a single-copy expression of an endogenous genomic acr-2 transgene (Frøkjaer-Jensen et al., 2008) in which GFP was inserted in the cytoplasmic loop between transmembrane domains TM3 and TM4 (oxSi39) (Fig. 1A). oxSi39 was fully functional, because it completely suppressed the spontaneous convulsive behaviors of $a c r-2(g f)$ animals and restored sinusoidal locomotion (Fig. $1 B, C$ ), indicating that the pattern and level of ACR-2::GFP expression should closely resemble endogenous ACR-2. Because live GFP fluorescence from oxSi39 was barely visible, we immunostained the animals using anti-GFP antibodies. ACR-2::GFP showed mostly diffused localization on neuronal soma, as well as processes in the ventral cord, sublateral cords, and nerve ring (Fig. 1D; data not shown). In the ventral cord, expression of ACR-2::GFP was specifically observed in the VA, VB, DA, and DB motor neurons as reported previously (Jospin et al., 2009). Several additional cells (e.g., SDQ, vulva muscles) also expressed ACR-2::GFP, presumably because of the use of a longer promoter and inclusion of intronic sequences (Fig. 1A). However, staining of ACR-2::GFP was not seen in the dorsal nerve cord (Fig. 1D). Because the axonal processes of DA and DB motor neurons are major constituents of the dorsal nerve cord, lack of ACR-2::GFP signals in the dorsal nerve cord indicates that ACR-2 is unlikely to localize to presynaptic sites of these motor neurons.

To further determine the precise localization pattern of ACR-2 in a single motor neuron, we expressed ACR-2::GFP specifically in DA9 neuron using Pitr-1 pB promoter (Gower et al., 2001). ACR-2::GFP signal was observed at DA9 soma, its entire dendritic branch, and the ventralmost proximal axonal region immediately posterior to soma (Fig. $1 E$ ). ACR-2::GFP staining was not detectable in the commissure or any part of the dorsal branch. Moreover, the dendritic ACR-2::GFP in DA9 did not show prominent clustering.

The dendritic processes of individual acetylcholine A and B motor neurons are not only postsynaptic to the premotor interneurons but are also in close proximity to the axonal release sites of neighboring motor neurons of the same class (White et al., 1986). To determine the spatial relationship of ACR-2 with respect to the presynaptic release sites of motor neurons in the ventral cord, we performed coimmunolabeling of ACR-2::GFP and UNC-17, the vesicular acetylcholine transporter that labels presynaptic termini of the all acetylcholine motor neurons (Duerr et al., 2008). ACR-2::GFP and UNC-17 staining patterns showed no significant overlap, or juxtapose, in the ventral cord (Fig. $1 F, G$ ). Moreover, the average distance between adjacent UNC-17 and ACR-2::GFP signals was $\sim 450 \mathrm{~nm}$ apart from each other (Fig. $1 F$ ). These data suggest that the majority of ACR-2 in $\mathrm{B}$ and $\mathrm{A}$ motor neurons are unlikely to function as classic post- 
A

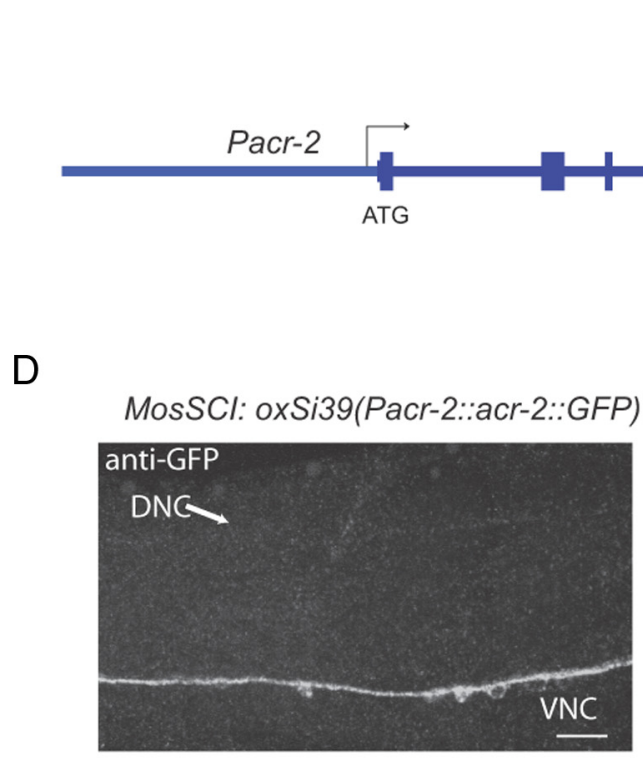

B

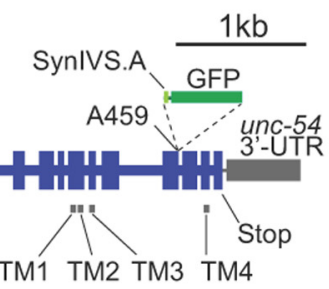

E

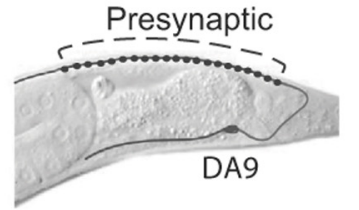

Ex[Pitr-1 pB-acr-2(wt)cDNA::GFP]

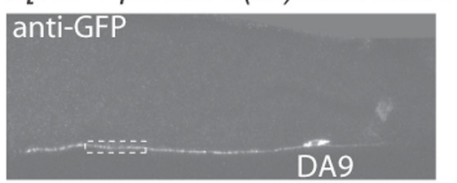

Ex[Pitr-1 pB-acr-2(gf)cDNA::GFP]

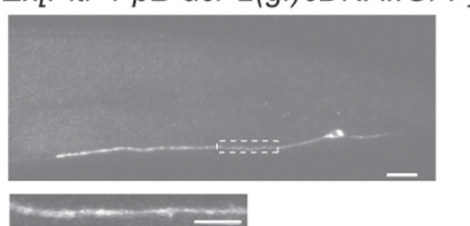

acr-2(gf); oxSi39

(Pacr-2::acr-2::GFP)
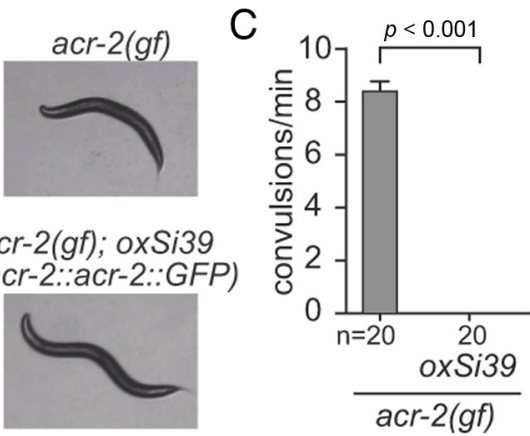

F

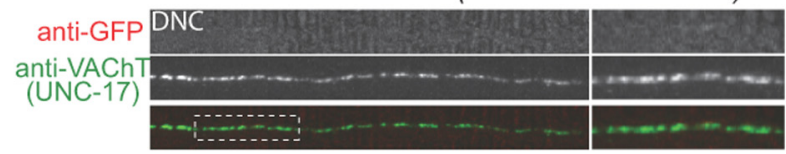

anti-GFP anti-VACh (UNC-17)
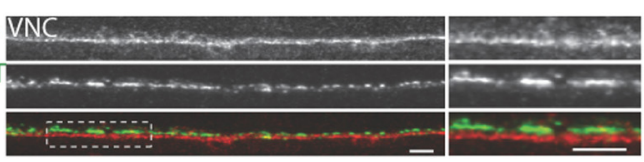

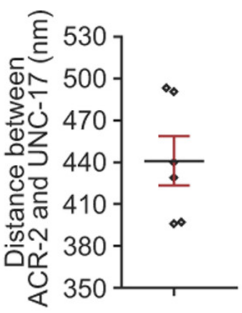

G

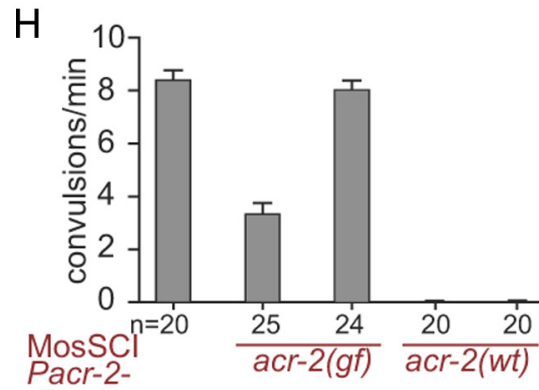

Back-acr-2(gf)wt acr-2(0) wt acr-2(0) ground

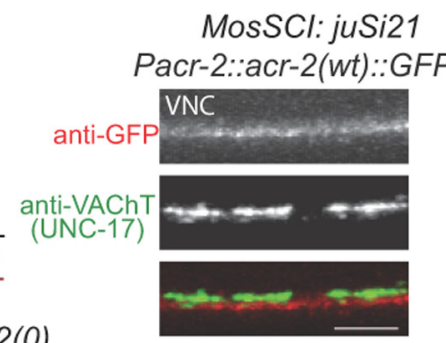

MosSCl: juSi19

Pacr-2::acr-2(gf)::GFP

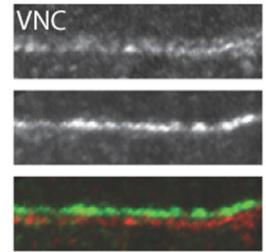

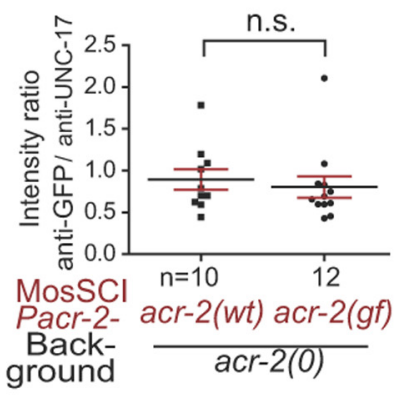

Figure 1. ACR-2 is localized dendritically in A/B-type motor neurons. $A$, The transgene construct for generating the single-copy genomic insertion allele oxSi39[Pacr-2::acr-2::GFP]. It contains genomic acr-2 sequence with gfp sequence inserted after the codon alanine 459. Expression from the construct is driven by $3.4 \mathrm{~kb}$ promoter. $\boldsymbol{B}$, Bright-field images showing that oxSi39 restored sinusoidal locomotion in acr-2(gf). C, Quantification showing that oxSi39 suppressed convulsions in acr-2(gf). D, Anti-GFP staining of the oxSi39 showing the ventral and dorsal nerve cord (VNC, DNC). The signals are primarily localized to the VNC but not the DNC. E, Anti-GFP staining of the animals carrying Pitr-1-pB-acr-2::gfp (left) and Pitr-1-pB-acr-2(gf)::gfp (right). The signals are only localized to the ventral branch and soma of DA9. The pictures in high magnification are shown below. $\boldsymbol{F}$, Double labeling using anti-GFP and anti-UNC-17. The DNC exhibits anti-UNC-17 but no anti-GFP signals. There is little overlapping of GFP and UNC-17 signals in the VNC. The pictures of high magnification are shown at the right side. Right shows the measured distance between the fluorescent spots with peak intensity for anti-GFP and anti-UNC-17. Each data point represents the average distance on individual images. The black line represents the average and red line represents SEM. G, Illustration summarizing the localization of ACR-2 on motor neurons. ACR-2 is localized to the dendritic branches of motor neurons, as well as cell bodies. H, Double labeling of anti-GFP and anti-UNC-17 in animals carrying Pacr-2-acr-2(CDNA)::gfp and Pacr-2-acr-2(gf)(CDNA):::gfp (middle). The left graph shows the convulsion rates of these animals. The right graph shows that the relative intensity of ACR-2::GFP signals are the same between acr-2(wt) and acr-2(gf) transgenes. Scale bars: $\mathbf{D}, 10 \mu \mathrm{m} ; \boldsymbol{E}, \boldsymbol{F}, \boldsymbol{H}, 5 \mu \mathrm{m}$. Statistics used two-tailed Student's test. Error bar shows SEM.

synaptic receptors, at least with respect to anatomically defined synaptic release sites.

\section{acr-2(gf) receptor does not show altered abundance}

\section{or localization}

acr-2(gf) mutation causes a valine to methionine change at the $13^{\prime}$ position of the pore-forming second transmembrane domain of ACR-2 (Jospin et al. 2009). acr-2(gf) mutants exhibit an increased acetylcholine MPSC at the NMJs and a hypersensitivity to the acetylcholine esterase inhibitor aldicarb (Jospin et al., 2009), implying increased ACh release. In vertebrates, membrane expression of nAChRs in the CNS can be upregulated when chronically exposed to nicotine (Buisson and Bertrand, 2001; Vallejo et al., 2005). To examine the possibility that elevated ACh levels in 
A
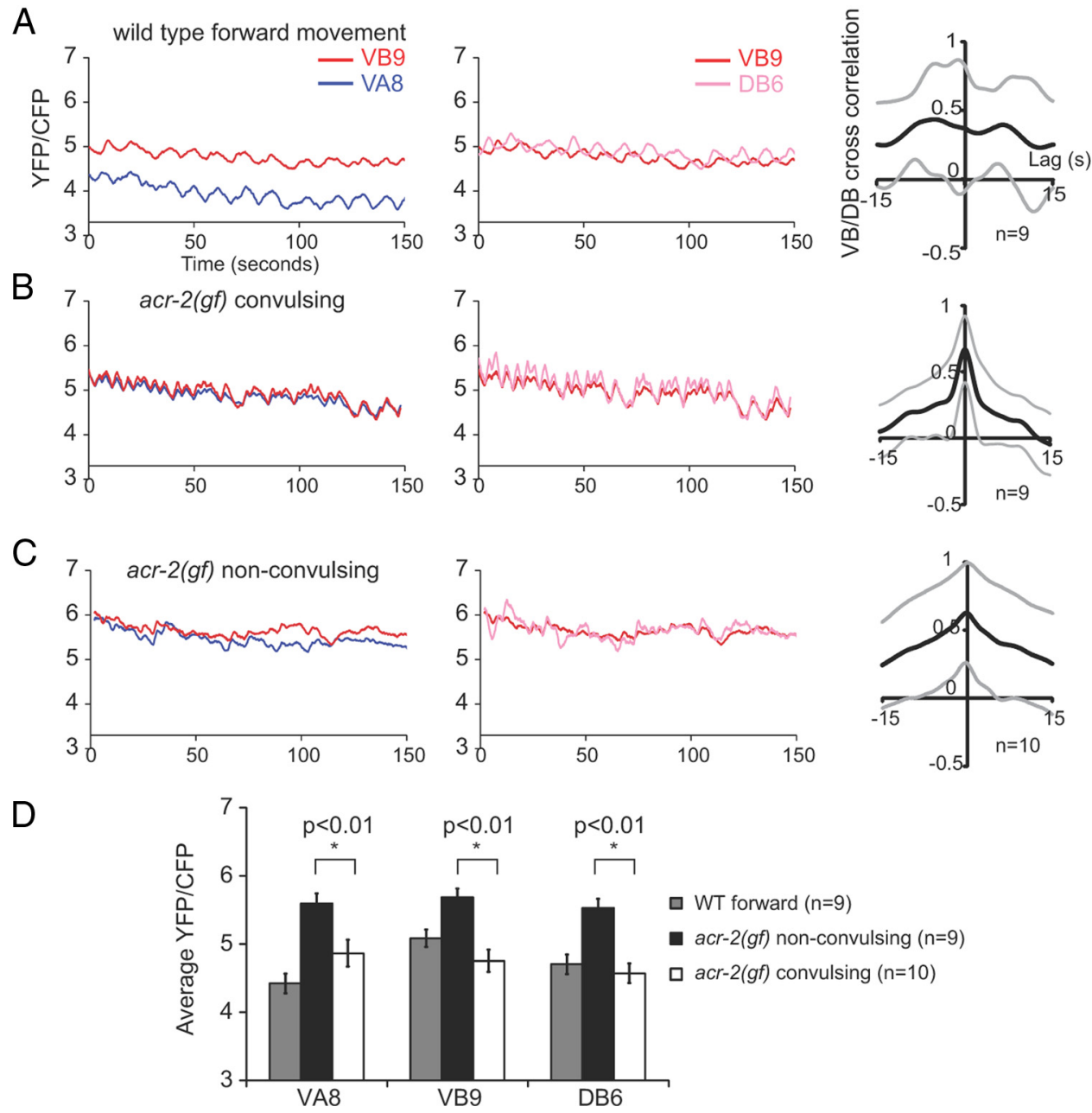

Figure 2. Convulsions of acr-2(gf) correspond to simultaneous excitatory motor neuron activity. Representative calcium imaging traces of VB9 versus VA8, VB9 versus DB6 motor neurons during forward movement of a wild-type animal $(\boldsymbol{A})$, a convulsing period of an acr-2(gf) animal $(\boldsymbol{B})$, and a nonconvulsing period of an acr-2( $g f$ ) animal $(\boldsymbol{C})$. In wild types, VB9 shows a higher calcium level than VA8 in accordance with forward locomotion; in convulsive acr-2(gf), VB9 exhibits the same calcium level as VA8. In wild types, VB9 and DB6 show alternating calcium peaks; in acr-2(gf), the alternation changed into frequent synchronization, corresponding to convulsion events. Right column show cross-correlations between VB9 and DB6 calcium signals. There are high correlations between VB9 and DB6 in acr-2(gf), especially during convulsions. Black lines represent the mean cross-correlation, and the gray lines represent 1 SD above and below the mean. Bar graph (D) showing average yellow fluorescent protein (YFP)/cyan fluorescent protein (CFP) ratio over the 150 s recording interval. When acr-2(gf) animals are not convulsing, VB9, VA8, and DB6 show similar calcium levels, which is significantly higher than when worms are convulsing. Statistics used two-tailed Student's $t$ test. Error bar shows SEM.

acr-2(gf) animals leads to an upregulated expression or an altered subcellular localization of the ACR-2(gf) receptors we created a single copy ACR-2(gf)(cDNA)::GFP transgene in acr-2 null animals. These animals exhibited a comparable convulsive behavior with that of the acr-2(gf) animals (Fig. $1 H)$. Immunostaining revealed that ACR-2(gf)::GFP signal showed neither an elevation nor an altered subcellular localization in reference to endogenous UNC-17 (Fig. 1H). Similarly, in the DA9 motor neuron, ACR-2(gf)::GFP showed a restricted expression pattern and level indistinguishable from that of the wild-type ACR-2::GFP (Fig. $1 E)$. Together, ACR-2(gf), which causes an increased ion conductance of ACh receptors reconstituted in oocytes (Boulin et al., 2008; Jospin et al., 2009), does not lead to changes to their dendritic localization or the expression level. Therefore, the convulsion behavior of acr-2(gf) animals is caused by the aberrant channel activity.

The motor output of acr-2(gf) animals reflects an aberrant synchrony of motor neuron activity

The sinusoidal locomotion pattern of wild-type animals is correlated with coordinated motor neuron activity such that there is a characteristic phase lag for the dorsal-ventral, anterior-posterior motor output (Kawano et al., 2011). To understand the cause of convulsive behavior of acr-2(gf), we examined the activity coordination between the $\mathrm{A}$ and $\mathrm{B}$ motor neurons using a calciumsensitive probe cameleon in moving animals (Kawano et al., 2011; Fig. 2). Similar to recent reports (Kawano et al., 2011; Wen et al., 2012), in wild-type animals, the B motor neurons that innervate the opposing ventral and dorsal body wall muscles, VB9 and DB6, respectively, exhibit an off-phase activation pattern (Fig. 2A). acr-2(gf) animals alternate between convulsive and nonconvulsive periods. During convulsions, VB9 and DB6 exhibited synchronized and equal levels of calcium transient changes (Fig. 2B). During the nonconvulsive periods, the VB9 and DB6 activity pattern was less synchronized but also failed to exhibit a significant off-phase correlation (Fig. 2C). Interestingly, the mean VB9 and DB6 calcium levels were lower during convulsions when compared with those during the nonconvulsive periods (Fig. 2D).

As reported, wild-type animals maintained a separation of the mean calcium transient levels between the A and B motor neurons that underlie directional movement: VB9 and VA8 exhibited 
A

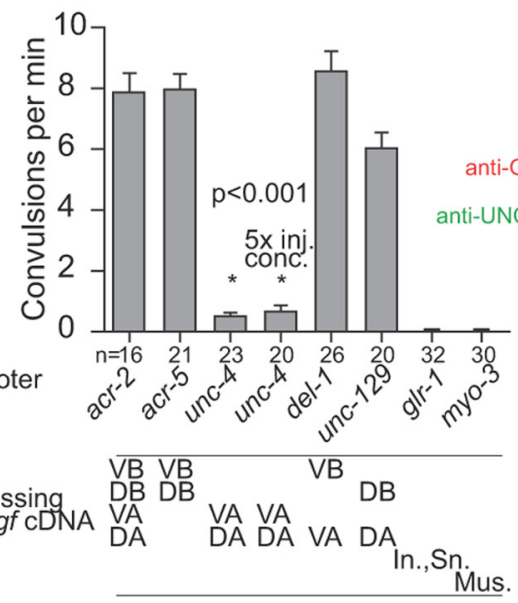

C

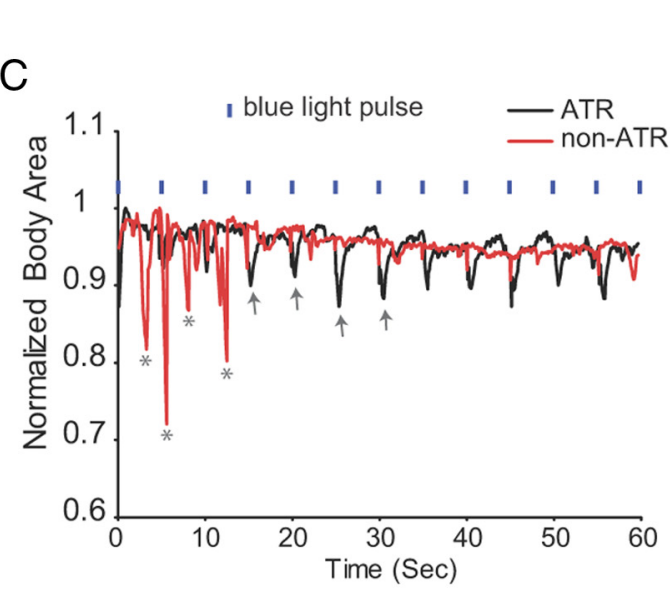

B

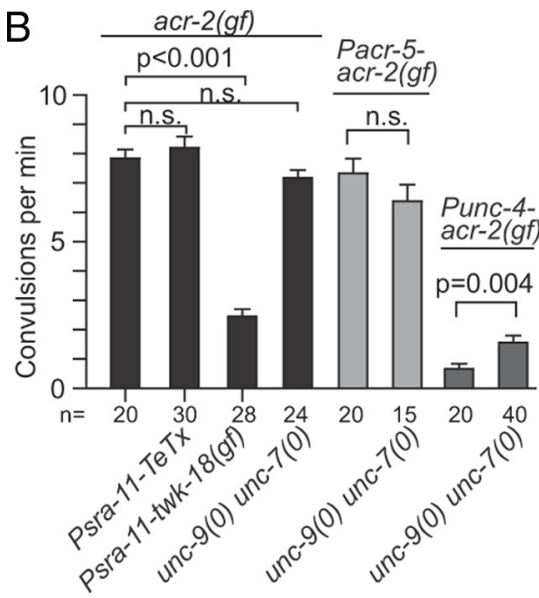

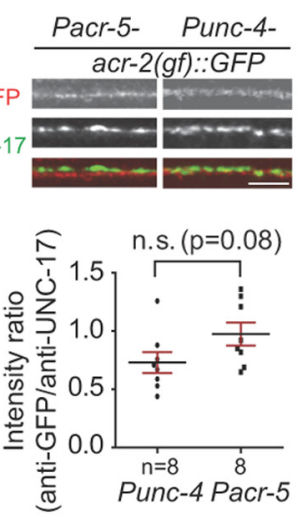

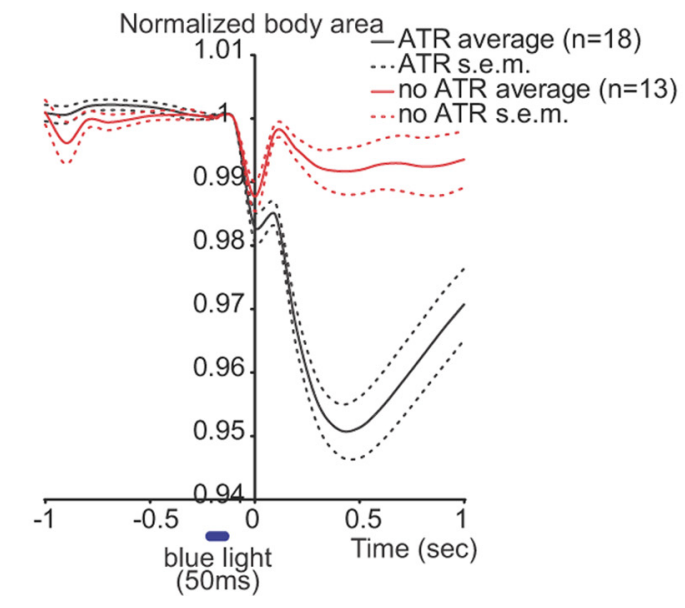

Figure 3. The excitation state of $B$ motor neurons is regulated by $A V B$ and is essential to trigger aberrant motor synchrony. $A$, Transgenic expression of $A C R-2$ (gf) by various promoters showed differential convulsive behaviors. ACR-2(gf) expression in A motor neurons was not able to induce convulsions, even at $5 \times$ injection concentration. Right shows that the Pacr-5 and Punc-4 transgenes have comparable expression of ACR-2(gf). In., Interneurons; Sn., sensory neurons. Mus., muscles. Scale bar, $5 \mu \mathrm{m}$. B, The convulsive behavior of $a c r-2$ (gf) was altered during AVB activity modulation by TeTx or TWK-18(gf). The loss of unc-7 and unc- 9 did not suppress convulsion frequency in acr-2(gf) or Pacr-5-acr-2(gf) transgene animals but weakly enhanced the convulsion in Punc-4-acr-2(gf) animals. C, ChR expressed in AVB induces convulsion in acr-2(gf). Left is a representative plot of the body areas of individual worms receiving blue light illumination. acr-2(gf) animals raised on ATR plates showed convulsions (arrows) during blue light, whereas control animals only showed spontaneous convulsions (asterisk). In the right, the average body areas of the worms are plotted before and after the blue light flashing. Statistics used two-tailed Student's $t$ test. Error bar shows SEM.

variable phase correlations; a VB9 > VA8 calcium transient pattern corresponds with forward locomotion and a VB9 $<$ VA8 pattern during backing (Kawano et al., 2011). This coordination was also disrupted in acr-2(gf) mutants (Fig. $2 A-C$ ). In acr-2(gf) mutants, during convulsions, VA8 and VB9 exhibited strictly synchronized and equal levels of calcium transient changes (Fig. $2 B$ ). During nonconvulsive periods, the mean activity level of VA8 and VB9 became more separated, and VA8 and VB9 neurons did not exhibit obvious phase correlation (Fig. 2C). Like the B motor neurons (VB9 and DB6), the A motor neuron (VA8) also exhibited a lower mean calcium transient level during convulsions when compared with that of the nonconvulsive periods (Fig. 2D). The lower calcium level during the convulsive periods may result from successive synchronous synaptic release, which is possibly analogous to short-term synaptic depression (Fioravante and Regehr, 2011).

Together, these data demonstrate that ACR-2(gf) aberrantly synchronizes the activation of motor neurons that innervate dorsal and ventral body wall muscles, as well as those that instruct forward and backward locomotion. Therefore, the hyperactivation of these motor neurons by somatodendritic ACR-2(gf) disrupts the ability of the motor circuit to establish an out-of-phase correlation between $\mathrm{VB}$ and $\mathrm{DB}$ activity and a differential activity level between the A- and B-class motor neurons.

\section{Dendritic hyperactivation of B class of motor neurons accounts for aberrant motor circuit synchrony}

We next investigated in which group of neurons in the motor circuit ACR-2(gf) drives motor circuit synchrony. We specifically expressed ACR-2(gf) in the subgroups of B and A motor neurons. Expressing ACR-2(gf) in the VB and DB neurons (Pacr-5) alone consistently induced the convulsive behavior (Fig. $3 A$ ). In contrast, when ACR-2(gf) was expressed in the VA and DA motor neurons (Punc-4), the transgenic animals did not show convulsive behaviors (Fig. $3 A$ ) and occasionally exhibited a coiled body and jerky backward movement. This differential effect was not attributable to an insufficient expression of the transgene because the anti-GFP immunostaining revealed comparable ACR-2(gf)::GFP expression levels in these transgenic animals (Fig. $3 A$ ).

To further test whether ACR-2(gf) functions through the ventral or dorsal subclasses of $\mathrm{B}$ motor neurons (VB or $\mathrm{DBs}$ ), we compared the effect of expressing ACR-2(gf) in VB and VA (Pdel-1) or in DB and DA (Punc-129). Both transgenes consis- 
tently induced specific convulsive behaviors (Fig. 3A): ACR-2(gf) expressed in VB and VA caused ventral convulsions, and ACR-2(gf) expressed in DB and DA caused dorsal convulsions, implying a simultaneous contraction of body wall muscles stimulated by corresponding ventral- or dorsal-innervating motor neurons. The effect of ACR-2(gf) was specific to motor neurons, because ACR-2(gf) expression in body wall muscles (Pmyo-3) or premotor interneurons $(P g l r-1)$ did not induce convulsions (Fig. $3 A$ ). Together, these data show that the hyperactivated state in B motor neuron by ACR-2(gf) is sufficient and necessary to induce the synchronized ventral and dorsal muscle contraction.

\section{AVB premotor interneurons potentiate $B$ motor neuron-dependent motor network synchrony}

$\mathrm{A}$ and $\mathrm{B}$ motor neurons receive the vast majority of chemical synaptic inputs from premotor interneurons AVA, AVE, AVD, and PVC (White et al., 1986), among which at least AVA, AVE, and PVC may release ACh (data not shown) (Duerr et al., 2008). However, expression of TeTx to inhibit chemical synaptic transmission (Schiavo et al., 1992) from these premotor interneurons (Pnmr-1) did not show any effect on ACR-2(gf)-mediated convulsions (data not shown), implying that ACR-2(gf) channels may receive acetylcholine released by other neurons, possibly ventral cord acetylcholine motor neurons (Fig. $1 G$ ).

The premotor interneuron AVBs make prominent gap junctions with B motor neurons (White et al., 1986). If the AVB neurons are ablated in $a c r-2(g f)$, using a genetically encoded free radical generator, the convulsions are essentially suppressed (Qi et al., 2012). This result indicates that the AVBs potentiate the ability of the B motor neurons to induce motor network synchronization. The effect of AVB on the B neurons is linked to its baseline level of excitation. $t w k-18(g f)$ encodes a hyperactivated outwardly rectifying $\mathrm{K}^{+}$channel, the overexpression of which is expected to hyperpolarize and consequently silence neurons (Kunkel et al., 2000; Kawano et al., 2011). When we expressed TWK-18(gf) in AVB, convulsions in acr-2(gf) were significantly decreased (Fig. 3B). In contrast, expressing TeTx to specifically block synaptic transmission in AVB did not reduce the frequency of convulsions in acr-2(gf) animals (Fig. $3 B$ ). Although the effectiveness of such a transgene could be a caveat, the results suggest that nonvesicular communication between AVB and B motor neurons likely plays a major role.

To determine the effect of AVB activation on the convulsive behavior of $a c r-2(g f)$, we expressed channelrhodopsin-2 (ChR2), a blue-light-gated cation channel in the presence of ATR (Nagel et al., 2005) in AVB. The convulsive events were quantified and defined by the sharp decrease of the body area (Fig. 3C), which results from whole-body contraction. Animals cultured with ATR consistently show convulsions during the light illumination (Fig. 3C). In contrast, convulsions of transgenic animals raised on non-ATR plates did not correlate with the occurrence of blue light illumination (Fig. 3 C). The activation of AVB is sufficient to induce aberrant synchronous activation of $\mathrm{B}$ motor neurons, hence the onset of convulsive event in $a c r-2(g f)$. Together, these data support the role of AVB in modulating B motor neuron activity.

UNC-7 and UNC-9 innexins reside at the AVB-B gap junctions (Starich et al., 2009). The loss of $u n c-7$ and $u n c-9$ did not decrease convulsions frequency of $a c r-2(g f)$ or convulsions caused by specific expression of ACR-2(gf) in B motor neurons (Fig. 3B), suggesting the existence of additional form(s) of AVB-B communication other than UNC-7- and UNC-9-mediated electrical coupling. The unc-7 and unc-9 mutations moderately enhanced the convulsive behavior induced by selective expression of ACR-2(gf) in A motor neurons (Fig. 3B), which may result from a hyperactivated backward circuit activity in $u n c-7$ and unc-9 mutants (Kawano et al., 2011). Together, the high activity of AVB leads to the potentiation of the B motor circuit via yet to be defined mode of AVB-B communication. Hyperpolarization of AVB by expressing twk-18(gf), a hyperactivated $\mathrm{K}^{+}$channel, is likely to depress $\mathrm{B}$ motor neurons and attenuate the aberrant synchronous circuit activity induced by $a c r-2(g f)$.

\section{Discussion}

Here, we have shown that the ionotropic acetylcholine receptor ACR-2 is localized to the dendrites and soma of A and B motor neurons (Fig. 4A). Our calcium imaging of the A and B motor neurons predicts that an in-phase activation of both ventraldorsal and anterior-posterior excitatory motor neurons leads to the synchronous activation of body wall muscles (Fig. 4B), which results in convulsions. An important implication of our findings is that there must normally be communications between ventral and dorsal motor neurons, as well as neighboring motor neurons of the same class. In wild-type animals, the activity of B motor neurons is proportional to the extent of body bends (Wen et al., 2012). Strikingly, hyperactivation of the B motor neurons alone impairs the propagation of sinusoidal body bends and directional movement, suggesting a dominant role of B motor neurons in the motor circuit.

In motor neurons, dendritic ACR-2 receptors do not show close apposition to presynaptic release sites, suggesting that they may act as extrasynaptic receptors to modulate motor circuit 
activity. The hyperactive ACR-2(gf) channel disrupts the phase alternation in motor circuit. Therefore, ACR-2 may be one of the substrates underlying wave propagation and the activity state of the motor circuit. Because acr-2(lf) animals do not exhibit obvious locomotion defects (Jospin et al., 2009), ACR-2-dependent extrasynaptic transmission may only exert limited influence on the motor circuit under physiological conditions or unidentified receptors may function redundantly with ACR-2. Indeed, dopamine signaling has been shown to dictate $C$. elegans velocity by activating extrasynaptic, antagonistic dopamine receptors expressed by these motor neurons (Chase et al., 2004). Because acr-2(gf) animals exhibit spontaneous convulsions, a unique phenotype among all known $C$. elegans neuronal activity-aberrant mutants, extrasynaptic transmission can exert a profound impact on the activity of the motor circuit. It is of interest to note that extrasynaptic action of neurotransmitters has been linked to the rhythmic activity of neural networks in vertebrate nervous systems (Scanziani, 2000; Blankenship et al., 2009). Our work provides an analogous system to study molecular substrates modulating circuit activity.

\section{References}

Bargmann CI (2012) Beyond the connectome: how neuromodulators shape neural circuits. BioEssays 34:458-465. CrossRef Medline

Blankenship AG, Ford KJ, Johnson J, Seal RP, Edwards RH, Copenhagen DR, Feller MB (2009) Synaptic and extrasynaptic factors governing glutamatergic retinal waves. Neuron 62:230-241. CrossRef Medline

Boulin T, Gielen M, Richmond JE, Williams DC, Paoletti P, Bessereau JL (2008) Eight genes are required for functional reconstitution of the Caenorhabditis elegans levamisole-sensitive acetylcholine receptor. Proc Natl Acad Sci U S A 105:18590-18595. CrossRef Medline

Buisson B, Bertrand D (2001) Chronic exposure to nicotine upregulates the human $\alpha 4 \beta 2$ nicotinic acetylcholine receptor function. J Neurosci 21: 1819-1829. Medline

Chalfie M, Sulston JE, White JG, Southgate E, Thomson JN, Brenner S (1985) The neural circuit for touch sensitivity in Caenorhabditis elegans. J Neurosci 5:956-964. Medline

Chase DL, Pepper JS, Koelle MR (2004) Mechanism of extrasynaptic dopamine signaling in Caenorhabditis elegans. Nat Neurosci 7:1096-1103. CrossRef Medline

de Bono M, Maricq AV (2005) Neuronal substrates of complex behaviors in C. elegans. Annu Rev Neurosci 28:451-501. CrossRef Medline

Duerr JS, Han HP, Fields SD, Rand JB (2008) Identification of major classes of cholinergic neurons in the nematode Caenorhabditis elegans. J Comp Neurol 506:398-408. CrossRef Medline

Fioravante D, Regehr WG (2011) Short-term forms of presynaptic plasticity. Curr Opin Neurobiol 21:269-274. CrossRef Medline

Frøkjaer-Jensen C, Davis MW, Hopkins CE, Newman BJ, Thummel JM, Olesen SP, Grunnet M, Jorgensen EM (2008) Single-copy insertion of transgenes in Caenorhabditis elegans. Nat Genet 40:1375-1383. CrossRef Medline

Gower NJ, Temple GR, Schein JE, Marra M, Walker DS, Baylis HA (2001) Dissection of the promoter region of the inositol 1,4,5-trisphosphate receptor gene, itr-1, in C. elegans: a molecular basis for cell-specific expression of IP3R isoforms. J Mol Biol 306:145-157. CrossRef Medline
Jospin M, Qi YB, Stawicki TM, Boulin T, Schuske KR, Horvitz HR, Bessereau JL, Jorgensen EM, Jin Y (2009) A neuronal acetylcholine receptor regulates the balance of muscle excitation and inhibition in Caenorhabditis elegans. PLoS Biol 7:e1000265. CrossRef Medline

Kawano T, Po MD, Gao S, Leung G, Ryu WS, Zhen M (2011) An imbalancing act: gap junctions reduce the backward motor circuit activity to bias $C$. elegans for forward locomotion. Neuron 72:572-586. CrossRef Medline

Kunkel MT, Johnstone DB, Thomas JH, Salkoff L (2000) Mutants of a temperature-sensitive two-P domain potassium channel. J Neurosci 20: 7517-7524. Medline

Li W, Feng Z, Sternberg PW, Xu XZ (2006) A C. elegans stretch receptor neuron revealed by a mechanosensitive TRP channel homologue. Nature 440:684-687. CrossRef Medline

McIntire SL, Jorgensen E, Kaplan J, Horvitz HR (1993) The GABAergic nervous system of Caenorhabditis elegans. Nature 364:337-341. CrossRef Medline

Nagel G, Brauner M, Liewald JF, Adeishvili N, Bamberg E, Gottschalk A (2005) Light activation of channelrhodopsin-2 in excitable cells of Caenorhabditis elegans triggers rapid behavioral responses. Curr Biol 15: 2279-2284. CrossRef Medline

Qi YB, Garren EJ, Shu X, Tsien RY, Jin Y (2012) Photo-inducible cell ablation in Caenorhabditis elegans using the genetically encoded singlet oxygen generating protein miniSOG. Proc Natl Acad Sci U S A 109:7499-7504. CrossRef Medline

Richmond JE, Jorgensen EM (1999) One GABA and two acetylcholine receptors function at the C. elegans neuromuscular junction. Nat Neurosci 2:791-797. CrossRef Medline

Scanziani M (2000) GABA spillover activates postsynaptic GABA(B) receptors to control rhythmic hippocampal activity. Neuron 25:673-681. CrossRef Medline

Schiavo G, Benfenati F, Poulain B, Rossetto O, Polverino de Laureto P, DasGupta BR, Montecucco C (1992) Tetanus and botulinum-B neurotoxins block neurotransmitter release by proteolytic cleavage of synaptobrevin. Nature 359:832-835. CrossRef Medline

Starich TA, Xu J, Skerrett IM, Nicholson BJ, Shaw JE (2009) Interactions between innexins UNC-7 and UNC-9 mediate electrical synapse specificity in the Caenorhabditis elegans locomotory nervous system. Neural Dev 4:16. CrossRef Medline

Vallejo YF, Buisson B, Bertrand D, Green WN (2005) Chronic nicotine exposure upregulates nicotinic receptors by a novel mechanism. J Neurosci 25:5563-5572. CrossRef Medline

Van Epps H, Dai Y, Qi Y, Goncharov A, Jin Y (2010) Nuclear pre-mRNA $3^{\prime}$-end processing regulates synapse and axon development in C. elegans. Development 137:2237-2250. CrossRef Medline

Walrond JP, Stretton AO (1985) Reciprocal inhibition in the motor nervous system of the nematode Ascaris: direct control of ventral inhibitory motoneurons by dorsal excitatory motoneurons. J Neurosci 5:9-15. Medline

Wen Q, Po MD, Hulme E, Chen S, Liu X, Kwok SW, Gershow M, Leifer AM, Butler V, Fang-Yen C, Kawano T, Schafer WR, Whitesides G, Wyart M, Chklovskii DB, Zhen M, Samuel AD (2012) Proprioceptive coupling within motor neurons drives $C$. elegans forward locomotion. Neuron 76:750-761. CrossRef Medline

White JG, Southgate E, Thomson JN, Brenner S (1976) The structure of the ventral nerve cord of Caenorhabditis elegans. Philos Trans R Soc Lond B Biol Sci 275:327-348. CrossRef Medline

White JG, Southgate E, Thomson JN, Brenner S (1986) The Structure of the nervous system of the nematode Caenorhabditis elegans. Philos Trans R Soc Lond B Biol Sci 314:1-340. CrossRef Medline 